\title{
?19
}

TI 2020-039/III

Tinbergen Institute Discussion Paper

\section{Zero-diagonality as a linear structure}

Jan R. Magnus ${ }^{1}$

Enrique Sentana ${ }^{2}$

${ }^{1}$ Department of Econometrics and Data Science, Vrije Universiteit Amsterdam and Tinbergen Institute, Amsterdam

2 CEMFI, Madrid 
Tinbergen Institute is the graduate school and research institute in economics of Erasmus University Rotterdam, the University of Amsterdam and Vrije Universiteit Amsterdam.

Contact: discussionpapers@tinbergen.nl

More TI discussion papers can be downloaded at https://www.tinbergen.nl

Tinbergen Institute has two locations:

Tinbergen Institute Amsterdam

Gustav Mahlerplein 117

1082 MS Amsterdam

The Netherlands

Tel.: +31(0)205984580

Tinbergen Institute Rotterdam

Burg. Oudlaan 50

3062 PA Rotterdam

The Netherlands

Tel.: +31(0)10408 8900 


\title{
Zero-diagonality as a linear structure
}

\author{
June 28, 2020
}

\author{
Jan R. Magnus \\ Department of Econometrics and Data Science, \\ Vrije Universiteit Amsterdam and Tinbergen Institute \\ Enrique Sentana \\ CEMFI
}

Abstract: A linear structure is a family of matrices that satisfy a given set of linear restrictions, such as symmetry or diagonality. We add to the literature on linear structures by studying the family of matrices where all diagonal elements are zero, and discuss two econometric examples where these results can be fruitfully applied.

JEL Classification: C65, C21, C32

Keywords: Diagonality, Networks, Restricted matrices, Structural vector autoregressions

Declaration of interest: None

Corresponding author: Enrique Sentana, CEMFI, Casado del Alisal 5, 28014 Madrid, Spain. E-mail: sentana@cemfi.es 


\section{Introduction}

A linear structure ( $L$-structure) is a family of matrices of given order that satisfy a specific set of linear restrictions. For square matrices (the most common case) examples are symmetry, skew-symmetry, (strict) lower triangularity, and diagonality. The general theory of $L$-structures was developed in Magnus (1988), hereafter M88, with applications to solving systems of equations and optimization involving patterned matrices. This theory also plays a role in the estimation of multivariate models in which linearly restricted matrices appear. The purpose of this note is to complement these results by investigating the properties of an $L$-structure not studied so far: zero-diagonal matrices.

Matrices with restricted or zero diagonals arise naturally in networks. Let $W$ denote the $n \times n$ adjacency matrix of a directed network. The $i j$-th element of $W$ indicates the existence and strength of the link going from unit $i$ to unit $j$. One of the most salient properties of an adjacency matrix is that all its diagonal elements are zero because loops (i.e., self-links) are not allowed in directed networks. Consequently, $W$ is a zero-diagonal matrix. Graham and de Paula (2020) contains an up-to-date list of applications and references.

Zero-diagonal matrices also arise in structural vector autoregressions. Consider the $n$-variate time-series process

$$
y_{t}=\Phi y_{t-1}+J \Psi \xi_{t}
$$

where $\xi_{t} \mid I_{t-1} \sim$ i.i.d. $\left(0, I_{n}\right), \Psi$ is a diagonal matrix whose elements contain the free scale of the structural shocks, while the columns of $J$, whose diagonal elements are normalized to 1, measure the relative effects of each of the structural shocks on all the observed variables. The diagonal elements of $J-I_{n}$ are zero. Lanne et al. (2017) provide sufficient conditions for the full identification of the shocks and the free elements of $J$ and $\Psi$ in non-Gaussian models.

In this note we discuss in Section 2 some general features of an $L$-structure and define the operator and basis for the vector space associated with zerodiagonality. In Section 3 we briefly review the $L$-structure of diagonal matrices. In Section 4 we develop the new $L$-structure of zero-diagonal matrices, and Section 5 relates this new structure to strict lower triangularity. All proofs are in the appendix. 


\section{Linear structures and basis matrices}

Consider a real $n \times n$ matrix $A=\left(a_{i j}\right)$, restricted by some linear constraints, for example $a_{i j}=a_{j i}$ (symmetry), $a_{i j}=-a_{j i}$ (skew-symmetry), or $a_{i i}=0$ (zero-diagonality). The collection of matrices $A$ of a given order that satisfy a specific set of linear restrictions constitutes an $L$-structure.

Let vec $A$ denote the $n^{2} \times 1$ vector containing the columns of $A$, one underneath the other, and let $\psi(A)$ denote the vector containing only the 'essential' elements of the $L$-structure. For example, for a symmetric or lower triangular matrix, $\psi(A)$ is the $n(n+1) / 2 \times 1$ vector containing the lower triangular elements of $A$, ordered as vec $A$ but with some elements removed; a vector commonly denoted by $\operatorname{vech}(A)$.

In this note three $L$-structures and their corresponding $\psi$-vectors will be considered, depending on whether $A$ is

- zero-diagonal $\left(a_{i i}=0\right): \psi_{o}(A)$ of dimension $m_{o}=n(n-1)$,

- diagonal $\left(a_{i j}=0\right.$ for $\left.i \neq j\right): \psi_{d}(A)$ of dimension $m_{d}=n$, and

- strictly lower triangular $\left(a_{i j}=0\right.$ for $\left.i \leq j\right): \psi_{l}(A)$

of dimension $m_{l}=n(n-1) / 2$.

When $n=3$ the relevant $\psi$-vectors are

$$
\psi_{o}(A)=\left(\begin{array}{l}
a_{21} \\
a_{31} \\
a_{12} \\
a_{32} \\
a_{13} \\
a_{23}
\end{array}\right), \quad \psi_{d}(A)=\left(\begin{array}{l}
a_{11} \\
a_{22} \\
a_{33}
\end{array}\right), \quad \psi_{l}(A)=\left(\begin{array}{l}
a_{21} \\
a_{31} \\
a_{32}
\end{array}\right) .
$$

Given $\psi(A)$, the corresponding basis matrices (denoted by $\Delta$ ) are defined implicitly by

- $\Delta_{o} \psi_{o}(A)=\operatorname{vec} A$ for any zero-diagonal matrix $A$,

- $\Delta_{d} \psi_{d}(A)=\operatorname{vec} A$ for any diagonal matrix $A$, and

- $\Delta_{l} \psi_{l}(A)=\operatorname{vec} A$ for any strictly lower triangular matrix $A$.

The basis matrices $\Delta_{i}$ are of order $n^{2} \times m_{i}(i=o, d, l)$, and for $n=3$ they 
take the form

$$
\Delta_{o}=\left(\begin{array}{cccccc}
0 & 0 & 0 & 0 & 0 & 0 \\
1 & 0 & 0 & 0 & 0 & 0 \\
0 & 1 & 0 & 0 & 0 & 0 \\
0 & 0 & 1 & 0 & 0 & 0 \\
0 & 0 & 0 & 0 & 0 & 0 \\
0 & 0 & 0 & 1 & 0 & 0 \\
0 & 0 & 0 & 0 & 1 & 0 \\
0 & 0 & 0 & 0 & 0 & 1 \\
0 & 0 & 0 & 0 & 0 & 0
\end{array}\right), \quad \Delta_{d}=\left(\begin{array}{lll}
1 & 0 & 0 \\
0 & 0 & 0 \\
0 & 0 & 0 \\
0 & 0 & 0 \\
0 & 1 & 0 \\
0 & 0 & 0 \\
0 & 0 & 0 \\
0 & 0 & 0 \\
0 & 0 & 1
\end{array}\right), \quad \Delta_{l}=\left(\begin{array}{lll}
0 & 0 & 0 \\
1 & 0 & 0 \\
0 & 1 & 0 \\
0 & 0 & 0 \\
0 & 0 & 0 \\
0 & 0 & 1 \\
0 & 0 & 0 \\
0 & 0 & 0 \\
0 & 0 & 0
\end{array}\right) .
$$

The three matrices share the property that each column contains one single 1 and zeros elsewhere. This is not true for all $\Delta$-matrices - not, for example, for the duplication matrix $D$, associated with symmetry.

The following properties hold for each of the three $L$-structures $L\left(\Delta_{i}\right)$.

Proposition 1. We have, for $i=o, d, l$,

(a) $\Delta_{i}$ has full column rank $m_{i}$,

(b) $\Delta_{i}^{\prime} \Delta_{i}=I_{m_{i}}$,

(c) $\Delta_{i}^{+}=\Delta_{i}^{\prime}$,

(d) $\Delta_{i}^{\prime} \operatorname{vec} A=\psi_{i}(A)$ for all $A$,

(e) $\Delta_{i} \Delta_{i}^{\prime}$ vec $A=\operatorname{vec} A$ for all $A \in L\left(\Delta_{i}\right)$,

(f) $\partial \operatorname{vec} A / \partial\left(\psi_{i}(A)\right)^{\prime}=\Delta_{i}$ for all $A \in L\left(\Delta_{i}\right)$.

Note that $(d)$ is valid for all $A$ because these basis matrices are effectively elimination matrices. In contrast, $(e)$ and $(f)$ are only valid within the chosen $L$-structure. In Sections 3 and 4 we also show the effect of $\Delta_{d} \Delta_{d}^{\prime}$ and $\Delta_{o} \Delta_{o}^{\prime}$ on a general matrix $A$.

Strict lower triangularity and diagonality were discussed in M88 (Chapters 6 and 7 ), but the zero-diagonal $L$-structure has not been discussed and will be our main interest. Since skew-symmetric matrices are zero-diagonal, all results on zero-diagonal matrices apply also to them.

\section{Diagonality}

We first review some properties of the diagonal $L$-structure and present some generalizations. The relevant $\Delta$-matrix can be written as

$$
\Delta_{d}^{\prime}=\left(E_{11}, E_{22}, \ldots, E_{n n}\right),
$$


where $E_{i i}$ is the $n \times n$ matrix with 1 in the $i$ th diagonal position and zeros elsewhere. We know that $\Delta_{d} \psi_{d}(A)=\operatorname{vec} A$ when $A$ is diagonal (the implicit definition), and that for any $A$, diagonal or not,

$$
\Delta_{d} \Delta_{d}^{\prime} \operatorname{vec} A=\Delta_{d} \psi_{d}(A)=\operatorname{vec}(\operatorname{dg}(A))
$$

see M88, Theorem 7.3(ii), where the matrix $\operatorname{dg}(A)$ is a transformation of the matrix $A$ containing only its diagonal elements. In contrast, the matrix $\operatorname{diag}(a)$ is a function of the vector $a$ and contains the components of $a$ on its diagonal. Thus,

$$
\psi_{d}(\operatorname{diag}(a))=a, \quad \operatorname{diag}\left(\psi_{d}(A)\right)=\operatorname{dg}(A) .
$$

Since the $\psi_{d}$ operator only affects the diagonal elements of $A$ we have $\psi_{d}(A)=$ $\psi_{d}\left(A^{\prime}\right)$ and hence $\Delta_{d}^{\prime} K=\Delta_{d}^{\prime}$, where $K$ is the $n^{2} \times n^{2}$ commutation matrix; see M88, Theorem 7.4(i).

The next result links the $\psi_{d}$ operator to the Hadamard (or element-byelement) product.

Proposition 2. Given two matrices $A$ and $B$ both of dimension $n \times n$, we have

$$
\Delta_{d}^{\prime}(A \otimes B) \Delta_{d}=\Delta_{d}^{\prime}(B \otimes A) \Delta_{d}=A \odot B=B \odot A,
$$

and in particular, for any two diagonal matrices $\Lambda_{1}$ and $\Lambda_{2}$,

$$
\Delta_{d}^{\prime}\left(\Lambda_{1} \otimes \Lambda_{2}\right) \Delta_{d}=\Delta_{d}^{\prime}\left(\Lambda_{2} \otimes \Lambda_{1}\right) \Delta_{d}=\Lambda_{1} \Lambda_{2}=\Lambda_{2} \Lambda_{1} .
$$

The first expression in Proposition 2 applies also to rectangular matrices $A$ and $B$, as long as $A$ and $B$ have the same order, but the $\Delta_{d}$ matrices that pre- and postmultiply will then have different orders.

We conclude this short review by presenting a generalization of Proposition 2 and Theorem 7.7(i) of M88.

Proposition 3. Let $M$ be a diagonal $n^{2} \times n^{2}$ matrix with diagonal elements $\mu_{11}, \mu_{12}, \ldots, \mu_{n n}$. Then,

$$
\Delta_{d}^{\prime} M \Delta_{d}=\operatorname{diag}\left(\mu_{11}, \mu_{22}, \ldots, \mu_{n n}\right)
$$

and

$$
\Delta_{d} \Delta_{d}^{\prime} M \Delta_{d}=M \Delta_{d}
$$




\section{Zero-diagonality}

The class of diagonal matrices and the class of zero-diagonal matrices are complements.

Proposition 4. The $n^{2} \times n^{2}$ matrix $\left(\Delta_{o}, \Delta_{d}\right)$ is orthogonal.

This implies that $\Delta_{o}^{\prime} \Delta_{d}=0$ and

$$
\Delta_{o} \Delta_{o}^{\prime}=I_{n^{2}}-\Delta_{d} \Delta_{d}^{\prime}
$$

a diagonal idempotent matrix of rank $n(n-1)$.

From the implicit definition we have $\Delta_{o} \Delta_{o}^{\prime} \operatorname{vec} A=\operatorname{vec} A$ for all zerodiagonal $A$, while for any $n \times n$ matrix $A$,

$$
\Delta_{o} \Delta_{o}^{\prime} \operatorname{vec} A=\operatorname{vec}(A-\operatorname{dg}(A)) .
$$

Next we study the matrix $\Delta_{o}^{\prime} K \Delta_{o}$, where $K$ is the commutation matrix. While $K$ has the effect $K \operatorname{vec} A=\operatorname{vec} A^{\prime}$ for any $A$, the matrix $\Delta_{o}^{\prime} K \Delta_{o}$ has the effect $\Delta_{o}^{\prime} K \Delta_{o} \psi_{o}(A)=\psi_{o}\left(A^{\prime}\right)$ for any zero-diagonal $A$. It plays the role of the commutation matrix for zero-diagonal matrices.

Proposition 5. The matrix $\Delta_{o}^{\prime} K \Delta_{o}$ is a symmetric permutation matrix of $\operatorname{order} n(n-1)$, and hence orthogonal.

Let $M$ be an $n^{2} \times n^{2}$ diagonal matrix, possibly but not necessarily of the form $M_{1} \otimes M_{2}$ where $M_{1}$ and $M_{2}$ are diagonal of order $n$.

Proposition 6. Let the diagonal $n^{2} \times n^{2}$ matrix $M$ have diagonal elements $\mu_{11}, \mu_{12}, \ldots, \mu_{n n}$. Then,

$$
\Delta_{o}^{\prime} M \Delta_{o}=\operatorname{diag}\left(\mu_{12}, \mu_{13}, \ldots, \mu_{n, n-1}\right), \quad \Delta_{o}^{\prime} M \Delta_{d}=0,
$$

and

$$
\Delta_{o} \Delta_{o}^{\prime} M \Delta_{o}=M \Delta_{o}
$$

In particular, for any $n \times n$ diagonal matrix $\Lambda$,

$$
\Delta_{o}^{\prime}\left(\Lambda \otimes I_{n}\right) \Delta_{o}=\Lambda \otimes I_{n-1}
$$

In the last expression, the order of the two diagonal matrices cannot be reversed in general. So it is not true that $\Delta_{o}^{\prime}\left(I_{n} \otimes \Lambda\right) \Delta_{o}=I_{n-1} \otimes \Lambda$, unless $\Lambda=I_{n}$. 
Proposition 7. If $K+M$ is nonsingular, then

$$
\left(\Delta_{o}, \Delta_{d}\right)^{\prime}(K+M)^{-1}\left(\Delta_{o}, \Delta_{d}\right)=\left(\begin{array}{cc}
\left(\Delta_{o}^{\prime}(K+M) \Delta_{o}\right)^{-1} & 0 \\
0 & \left(I+\Delta_{d}^{\prime} M \Delta_{d}\right)^{-1}
\end{array}\right)
$$

and

$$
(K+M)^{-1}=\Delta_{o}\left(\Delta_{o}^{\prime}(K+M) \Delta_{o}\right)^{-1} \Delta_{o}^{\prime}+\Delta_{d}\left(I+\Delta_{d}^{\prime} M \Delta_{d}\right)^{-1} \Delta_{d}^{\prime}
$$

We can be more precise about the nonsingularity of $K+M$. Let $A_{i j}=$ $\alpha E_{i j}+\beta E_{j i}$. Then,

$$
(K+M) \operatorname{vec} A_{i j}=\operatorname{vec}\left(\alpha \mu_{i j}+\beta\right) E_{i j}+\operatorname{vec}\left(\alpha+\beta \mu_{j i}\right) E_{j i}=\lambda_{i j} \operatorname{vec} A_{i j}
$$

if and only if

$$
\alpha \mu_{i j}+\beta=\alpha \lambda_{i j}, \quad \alpha+\beta \mu_{j i}=\beta \lambda_{i j} .
$$

Hence, the eigenvalues of $K+M$ are $\lambda_{i i}=1+\mu_{i i}, \lambda_{i j}=p_{i j}+q_{i j}(i>j)$, and $\lambda_{i j}=p_{i j}-q_{i j}(i<j)$, where

$$
p_{i j}=\frac{\mu_{i j}+\mu_{j i}}{2}, \quad q_{i j}=\sqrt{\left(\frac{\mu_{i j}-\mu_{j i}}{2}\right)^{2}+1} .
$$

The matrix is nonsingular if and only if $\mu_{i i} \neq-1(i=1, \ldots, n)$ and $\mu_{i j} \mu_{j i} \neq 1$ for $i \neq j$.

\section{Zero-diagonality and strict lower triangu- larity}

The classes of zero-diagonal and strictly lower triangular matrices are also related, which can be exploited in several directions. Here we only mention the following result.

Proposition 8. The $n(n-1) \times n(n-1)$ matrix $\Delta_{o}^{\prime}\left(\Delta_{l}, K \Delta_{l}\right)$ is orthogonal, and

$$
\left(\begin{array}{c}
\Delta_{l}^{\prime} \\
\Delta_{l}^{\prime} K
\end{array}\right) \Delta_{o} \psi_{o}(A)=\left(\begin{array}{c}
\psi_{l}(A) \\
\psi_{l}\left(A^{\prime}\right)
\end{array}\right)
$$

This result is analogous to Theorem 7.5 in M88, which explains how to transform $\psi_{l}(A)$ and $\psi_{d}(A)$ jointly into vech $(A)$. 


\section{Appendix: Proofs}

Proof of Proposition 1: This follows from M88, Theorems 2.3 and 2.4. Properties (a), (e), and (f) are valid for all basis matrices. Properties (b) and (d) follow from the fact that the columns of our three basis matrices are selected columns of the identity matrix. Property (c) follows from (b).

Proof of Proposition 2: See M88, Theorem 7.7(ii).

Proof of Proposition 3: Let $\Delta_{d}^{\prime}=\left(E_{11}, E_{22}, \ldots, E_{n n}\right), e_{i}$ the $i$ th elementary vector so that $E_{i i}=e_{i} e_{i}^{\prime}$, and $M_{i}$ the $i$ th diagonal block (of order $n \times n$ ) of $M$. Then,

$$
\begin{aligned}
\Delta_{d}^{\prime} M \Delta_{d} & =\sum_{i=1}^{n} E_{i i} M_{i} E_{i i}=\sum_{i=1}^{n} e_{i} e_{i}^{\prime} M_{i} e_{i} e_{i}^{\prime}=\sum_{i=1}^{n} \mu_{i i} E_{i i} \\
& =\operatorname{diag}\left(\mu_{11}, \mu_{22}, \ldots, \mu_{n n}\right) .
\end{aligned}
$$

To prove the second equation, write $M=\sum_{i j} \mu_{i j}\left(E_{i i} \otimes E_{j j}\right)$ and let $A$ be diagonal. Then,

$$
\begin{aligned}
& \Delta_{d} \Delta_{d}^{\prime} M \Delta_{d} \psi_{d}(A)=\sum_{i j} \mu_{i j} \Delta_{d} \Delta_{d}^{\prime}\left(E_{i i} \otimes E_{j j}\right) \Delta_{d} \psi_{d}(A) \\
& =\sum_{i j} \mu_{i j} \Delta_{d} \Delta_{d}^{\prime}\left(E_{i i} \otimes E_{j j}\right) \operatorname{vec} A=\sum_{i j} \mu_{i j} \Delta_{d} \Delta_{d}^{\prime} \operatorname{vec}\left(E_{j j} A E_{i i}\right) \\
& =\sum_{i j} \mu_{i j} \operatorname{vec}\left(E_{j j} A E_{i i}\right)=\sum_{i j} \mu_{i j}\left(E_{i i} \otimes E_{j j}\right) \operatorname{vec} A \\
& =\sum_{i j} \mu_{i j}\left(E_{i i} \otimes E_{j j}\right) \Delta_{d} \psi_{d}(A)=M \Delta_{d} \psi_{d}(A),
\end{aligned}
$$

where we have used the diagonality of $E_{j j} A E_{i i}$. Since this holds for all $\psi_{d}(A)$, the proof is complete.

Proof of Proposition 4: Since the matrices $\Delta_{d}$ and $\Delta_{o}$ select the diagonal and nondiagonal elements of vec $A$, respectively, the matrix $\left(\Delta_{o}, \Delta_{d}\right)$ is a permutation matrix, hence orthogonal.

Proof of Proposition 5: Since $\Delta_{o}^{\prime} K \Delta_{o} \psi_{o}(A)=\psi_{o}\left(A^{\prime}\right)$ for any zerodiagonal $A$, the matrix $\Delta_{o}^{\prime} K \Delta_{o}$ is a permutation matrix. It is symmetric because $K$ is symmetric. Since all permutation matrices are orthogonal, the result follows. 
Proof of Proposition 6: As in the proof of Proposition 3, write $M=$ $\sum_{i j} \mu_{i j}\left(E_{i i} \otimes E_{j j}\right)$. Then,

$$
\begin{aligned}
\Delta_{o}^{\prime} M \Delta_{o} & =\sum_{i j} \mu_{i j} \Delta_{o}^{\prime}\left(E_{i i} \otimes E_{j j}\right) \Delta_{o}=\sum_{i j} \mu_{i j} \Delta_{o}^{\prime}\left(e_{i} \otimes e_{j}\right)\left(e_{i} \otimes e_{j}\right)^{\prime} \Delta_{o} \\
& =\sum_{i j} \mu_{i j} \psi_{o}\left(E_{j i}\right) \psi_{o}\left(E_{j i}\right)^{\prime}=\operatorname{diag}\left(\mu_{12}, \mu_{13}, \ldots, \mu_{n, n-1}\right) .
\end{aligned}
$$

Similarly,

$$
\Delta_{o}^{\prime} M \Delta_{d}=\sum_{i j} \mu_{i j} \psi_{o}\left(E_{j i}\right) \psi_{d}\left(E_{j i}\right)^{\prime}=0
$$

because either $\psi_{o}\left(E_{j i}\right)=0$ or $\psi_{d}\left(E_{j i}\right)=0$.

The proof of the third statement is the mirror image of the corresponding statement in Proposition 3. The special case $M=\Lambda \otimes I_{n}$ follows by noting that $\mu_{i j}=\lambda_{i}$ for all $j$, so that

$$
\mu_{12}, \mu_{13}, \ldots, \mu_{n, n-1}=\underbrace{\lambda_{1}, \ldots, \lambda_{1}}_{n-1 \text { times }}, \underbrace{\lambda_{2}, \ldots, \lambda_{2}}_{n-1 \text { times }}, \ldots, \underbrace{\lambda_{n}, \ldots, \lambda_{n}}_{n-1 \text { times }} .
$$

Proof of Proposition 7: We have

$$
\begin{gathered}
\left(\Delta_{o}, \Delta_{d}\right)^{\prime}(K+M)\left(\Delta_{o}, \Delta_{d}\right)=\left(\begin{array}{cc}
\Delta_{o}^{\prime}(K+M) \Delta_{o} & \Delta_{o}^{\prime} K \Delta_{d}+\Delta_{o}^{\prime} M \Delta_{d} \\
\Delta_{d}^{\prime} K \Delta_{o}+\Delta_{d}^{\prime} M \Delta_{o} & \Delta_{d}^{\prime} K \Delta_{d}+\Delta_{d}^{\prime} M \Delta_{d}
\end{array}\right) \\
=\left(\begin{array}{cc}
\Delta_{o}^{\prime}(K+M) \Delta_{o} & 0 \\
0 & I+\Delta_{d}^{\prime} M \Delta_{d}
\end{array}\right),
\end{gathered}
$$

using the facts that $K \Delta_{d}=\Delta_{d}$ (M88, Theorem 7.4(i)), $\Delta_{d}^{\prime} \Delta_{d}=I$ (Proposition 1), $\Delta_{d}^{\prime} \Delta_{o}=0$ (Proposition 4), and $\Delta_{d}^{\prime} M \Delta_{o}=0$ (Proposition 6). Since $\left(\Delta_{o}, \Delta_{d}\right)$ is orthogonal, the results follow.

Proof of Proposition 8: We have

$$
\begin{aligned}
\left(\begin{array}{c}
\Delta_{l}^{\prime} \\
\Delta_{l}^{\prime} K
\end{array}\right) & \Delta_{o} \Delta_{o}^{\prime}\left(\Delta_{l}, K \Delta_{l}\right)=\left(\begin{array}{c}
\Delta_{l}^{\prime} \\
\Delta_{l}^{\prime} K
\end{array}\right)\left(I_{n^{2}}-\Delta_{d} \Delta_{d}^{\prime}\right)\left(\Delta_{l}, K \Delta_{l}\right) \\
& =\left(\begin{array}{c}
\Delta_{l}^{\prime} \\
\Delta_{l}^{\prime} K
\end{array}\right)\left(\Delta_{l}, K \Delta_{l}\right)-\left(\begin{array}{c}
\Delta_{l}^{\prime} \\
\Delta_{l}^{\prime} K
\end{array}\right) \Delta_{d} \Delta_{d}^{\prime}\left(\Delta_{l}, K \Delta_{l}\right) \\
& =\left(\begin{array}{cc}
\Delta_{l}^{\prime} \Delta_{l}-\Delta_{l}^{\prime} \Delta_{d} \Delta_{d}^{\prime} \Delta_{l} & \Delta_{l}^{\prime} K \Delta_{l}-\Delta_{l}^{\prime} \Delta_{d} \Delta_{d}^{\prime} K \Delta_{l} \\
\Delta_{l}^{\prime} K \Delta_{l}-\Delta_{l}^{\prime} K \Delta_{d} \Delta_{d}^{\prime} \Delta_{l} & \Delta_{l}^{\prime} K K \Delta_{l}-\Delta_{l}^{\prime} K \Delta_{d} \Delta_{d}^{\prime} K \Delta_{l}
\end{array}\right)=I_{n^{2}}
\end{aligned}
$$

because $K$ is orthogonal, $\Delta_{l}^{\prime} \Delta_{l}=I_{n(n-1) / 2}$, and $\Delta_{l}^{\prime} K \Delta_{l}=0$ and $\Delta_{l}^{\prime} \Delta_{d}=0$ by virtue of Theorems 6.9 (iv) and $7.4(\mathrm{v})$ in M88, respectively. 
Next, let $A$ be zero-diagonal. Then,

$$
\begin{array}{r}
\left(\begin{array}{c}
\Delta_{l}^{\prime} \\
\Delta_{l}^{\prime} K
\end{array}\right) \Delta_{o} \psi_{o}(A)=\left(\begin{array}{c}
\Delta_{l}^{\prime} \\
\Delta_{l}^{\prime} K
\end{array}\right) \operatorname{vec} A \\
=\left(\begin{array}{c}
\Delta_{l}^{\prime} \operatorname{vec} A \\
\Delta_{l}^{\prime} \operatorname{vec} A^{\prime}
\end{array}\right)=\left(\begin{array}{c}
\psi_{l}(A) \\
\psi_{l}\left(A^{\prime}\right)
\end{array}\right) .
\end{array}
$$

Since the zero-diagonality of $A$ does not restrict $\psi_{o}(A), \psi_{l}(A)$, and $\psi_{l}\left(A^{\prime}\right)$, the result follows.

\section{Acknowledgements}

We are grateful to Gabriele Fiorentini for useful comments and discussions. Sentana gratefully acknowledges financial support from the Spanish Government through grant ECO 2017-89689.

\section{References}

Graham, B. and de Paula, A. (eds.) (2020). The Econometric Analysis of Network Data. Academic Press.

Lanne, M., Meitz, M. and Saikkonen, P. (2017). Identification and estimation of non-Gaussian structural vector autoregressions. Journal of Econometrics, 196, 288-304.

Magnus, J. R. (1988). Linear structures. Oxford University Press. 